\title{
MIEUX CONNAÎTRE LE NDAKINNA : LA CONTRIBUTION DE L'ARCHÉOLOGIE COLLABORATIVE AU PROCESSUS D'AFFIRMATION TERRITORIALE DE LA NATION ABÉNAKISE
}

\section{Conhecer melhor o Ndakinna: a contribuição da arqueologia colaborativa no processo de afirmação territorial da Nação Abénakise}

\author{
Geneviève Treyvaud * \\ Suzie O'Bomsawin ** \\ Marie-̇̀ve Samson ${ }^{* * *}$
}

\begin{abstract}
RÉSUMÉ
Les Abénakis, «les gens du pays de l'aube » ou « ceux qui vivent au levant », forment la Première Nation algonquienne la plus au sud du Québec. Le Ndakinna est leur territoire ancestral. Pour les Abénakis, le territoire est un espace qui inclut l'occupation physique, l'utilisation des ressources et la culture. Cependant, les informations sur l'occupation humaine du Ndakinna sont méconnues. Depuis 1979, le Grand Conseil de la Nation WabanAki, mandaté par le Conseil des Abénakis d'Odanak et le Conseil des Abénakis de Wôlinak, a comme mission d'assurer un avenir à la Nation Abénakise. Par le biais d'études spécialisées le Grand Conseil de la Nation Waban-Aki s'assure de la défense des droits, des intérêts et de la préservation de la culture de la Nation.
\end{abstract}

Mots-clés: Abénakis; territoire; archéologie; culture; valorisation; gestion

\footnotetext{
* Archéologue au Grand Conseil de la Nation Waban-Aki.

E-mail: gtreyvaud@gcnwa.com

** Directrice du Bureau du Ndakinna au Grand Conseil de la Nation Waban-Aki

*** Agente de recherche au Bureau du Ndakinna au Grand Conseil de la Nation Waban-Aki

Grand Conseil de la Nation Waban-Aki. 10175 Kolipaio. Wôlinak, Québec, CANADA. G0X 1B0
} 


\begin{abstract}
The Ndakinna is the ancestral territory of the Abenaki. For Abenaki territory is a space which includes its occupation, resource use and culture. The Abenaki "People of the dawn" are Québec's southernmost Algonkian First Nation. However, information on the occupation of Ndakinna is poorly understood. Since 1979, the Grand Council of the Waban-Aki Nation (GCNWA), mandated by the Councils of the Abenakis of Odanak and Wôlinak, has as its mission the future of the Abenaki. In undertaking specialist studies, the GCNWA, defends the Nation's rights, interests and the preservation of their culture.
\end{abstract}

Keywords: Abenaki; territory; archaeology; culture; development; management

\title{
RESUMO
}

O povo Abénaki, "aqueles do pais do sol nascente", constitui a Primeira Nação algonquina localizada no Sul da província canadense do Quebec. Ndakinna é o território ancestral dos Abenakis. Eles consideram o território como um espaço incluindo a ocupação física, o uso dos recursos e a cultura. Os dados sobre a ocupação humana do Ndakinna permanecem, porém, pouco conhecidos. Desde 1979, o Grande Conselho da Naçao WabanAki, habilitado pelo Conselho dos Abénakis de Odanak e o Conselho dos Abénakis de Wôlinak, tem como missão garantir o futuro da Nação Abénaki. Para este fim, o Grande Conselho usa estudos especializados para garantir a defesa dos direitos, dos interesses e a preservação da cultura da Nação.

Palavras-chave: Abenaki; territory; archaeology; culture; development; management

\section{Introduction}

Le Ndakinna («Notre territoire » en langue abénakise) est le territoire ancestral des Abénakis. Pour les Abénakis, le territoire ne se limite pas à une dimension physique. Il inclut autant l'occupation physique, l'utilisation des ressources qu'une dimension culturelle. Cette dernière est axée notamment sur le bien-être, la spiritualité, le maintien de relations sociales entre les humains et aussi avec les non- 
humains ainsi que la transmission de savoirs aux générations actuelles et futures (Clermont, 1980 ; Speck, 1998). Or, nous possédons très peu de connaissances sur la présence ancienne des Abénakis au Québec et sur l'occupation et l'utilisation du Ndakinna. Selon les sources historiques, la Confédération Wabanaki, « les gens du pays de l'aube » ou «ceux qui vivent au levant » regroupe les Premières Nations vivant sur un vaste territoire s'étendant de la côte de l'Atlantique incluant le Québec (Canada), le Maine et le Vermont (États-Unis). En général la communauté archéologique s'entend sur le fait qu'ils sont les descendants des peuples paléohistoriques du sylvicole (3000 AA à 1534 de notre ère) occupant cette région à l'arrivée des Européens au milieu du seizième siècle (figure 1). Les premiers récits historiques dressés par les Européens font état de familles abénakises qui vivaient en bourgades le long de cours d'eau l'été et se retiraient vers les forêts l'hiver. La Nation abénakise empruntait au rythme des saisons les réseaux hydrographiques situés entre la côte Atlantique et le fleuve Saint-Laurent afin de bénéficier de ressources naturelles diversifiées et de favoriser les échanges de biens avec d'autres groupes autochtones, ou encore avec les Européens. La Guerre du Roi Philippe en 1675 et 1676 provoque un exode des groupes abénakis du Maine et du Vermont vers le Québec. Certaines familles rejoignent les communautés abénakises du Québec et d'autres rejoignent les communautés malécites ou mi'kmaq. Au début du dix-huitième siècle et jusqu'à aujourd'hui, Odanak et Wôlinak, sur les rivières Saint-François et Bécancour deviennent les plus grands établissements abénakis dans le Nord-Est canadien (Calloway, 1994 ; Charland, 1964 ; Day, 1978 ; Treyvaud et Plourde, 2013, 2017). 
Figure 1 Carte du territoire ancestral abénaki

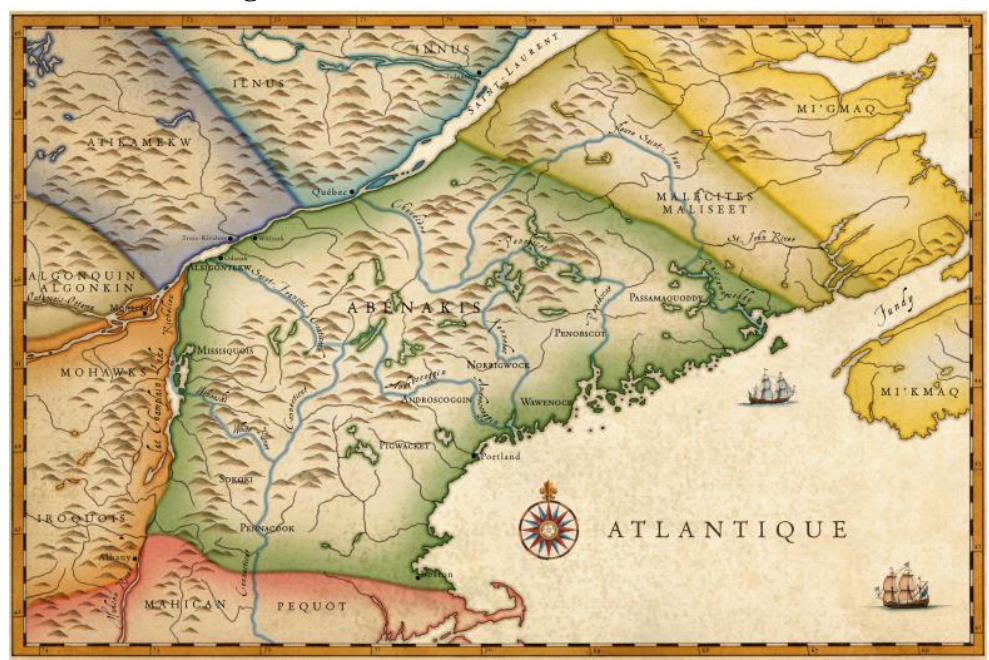

(C) Luc Normandin/Musée des Abénakis

Depuis 1979, le Grand Conseil de la Nation Waban-Aki, mandaté par les deux Conseils des Abénakis d'Odanak et de Wôlinak, constitue une entité administrative qui offre certaines ressources et services pour répondre à des besoins communs des deux communautés. Sa mission est déclinée en trois volets: la représentation, le développement et l'administration. En 2013, dans une volonté d'affirmer la présence de la Nation sur le territoire et face à l'augmentation considérable des demandes de consultation adressées à la Nation, le Bureau du Ndakinna a été créé. Avant sa création, les consultations et la gestion du territoire étaient gérées par les Conseils séparément, et ce de manière non systématique. Or, le nombre grandissant de consultations en lien avec le développement du territoire rendait de plus en plus ardue la défense des droits et intérêts de la Nation sur le Ndakinna. Le Bureau du Ndakinna est venu répondre à ce besoin en assumant un rôle de porte-parole en matière de questions territoriales ainsi que de défenseur et de promoteur des droits et des intérêts des Abénakis. Dans le contexte de création du Bureau, la recherche multidisciplinaire et collaborative, 
tant sur les plans historique, archéologique, anthropologique qu'environnemental (biologie et foresterie), s'est imposée comme un outil privilégié pour acquérir les connaissances nécessaires à une gestion du territoire selon une approche abénakise, et garantit dans le même temps une meilleure affirmation de la Nation sur le Ndakinna.

Il semblait ainsi tout naturel d'intégrer l'archéologie à ce processus, et ce, dès la création du Bureau du Ndakinna. Effectivement, plusieurs travaux archéologiques ont été effectués ou sont en cours de réalisation. Le projet «Fort Odanak : le passé revisité », «Wôlinak : La mission Saint-François Xavier (Musée des Abénakis et Patrimoine canadien)》 ainsi qu'une surveillance des travaux d'aménagement $\mathrm{du}$ territoire ont ainsi donné et génèrent encore des résultats archéologiques (TREYVAUD et PLOURDE, 2010, 2014, 2014, 2017). C'est pour cette raison que l'archéologue Geneviève Treyvaud et la directrice du Bureau du Ndakinna Suzie O'Bomsawin ont proposé d'intégrer l'ensemble des données archéologiques au projet général d'affirmation territoriale, de reconnaissance et de préservation du Ndakinna, qui est au cœur de la mission du Bureau du Ndakinna. Ce principe définit l'étendue du Ndakinna afin de permettre la protection et la promotion des droits et intérêts de la Nation dans un esprit de collaboration avec les différents intervenants présents sur son territoire, et ce, sans passer jusqu'alors par un processus judiciaire.

Dans cette perspective, les intervenants (biologiste, historien, géomaticien, archéologue et anthropologue) du Bureau du Ndakinna au GCNWA travaillent activement depuis mars 2013 à documenter et mieux comprendre l'utilisation et l'occupation du territoire de la Nation. Le Bureau du Ndakinna a d'abord mené une recherche historique sur la territorialité abénakise qui a contribué à mieux cerner les limites du territoire. Puis, à partir de 2014, le Bureau a entrepris une étude sur l'utilisation et l'occupation contemporaine du Ndakinna afin de comprendre l'importance de faire perdurer un mode de vie abénaki et de transmettre les savoirs associés au territoire pour la Nation. Cette étude a permis de mieux concevoir comment les Abénakis se représentent le Ndakinna et comment ils font face aux nombreuses transformations qui s'y opèrent actuellement.

Les résultats archéologiques obtenus documentent quant à eux les établissements abénakis et les ressources naturelles aux 
périodes préhistoriques, de contact et historiques et caractérisent leurs changements à travers le temps en mesurant l'impact de l'arrivée des Européens sur le Ndakinna. Ces projets d'archéologie sont conçus en un partenariat entre différentes institutions d'enseignement supérieur (l'Institut National des Recherches Scientifiques (INRS), unité Eau Terre Environnement et l'Université Laval), le Grand Conseil de la Nation Waban-Aki, les Conseils des Abénakis de Wôlinak et d'Odanak et les membres de la Nation abénakise.

Le choix d'une approche multidisciplinaire et collaboratrice est pertinent parce qu'il permet de construire les connaissances tant sur des savoirs acquis dans un milieu universitaire qu'au sein de la Nation, à travers les savoirs et les expériences du territoire des Abénakis. Le bureau du Ndakinna se veut rassembleur en proposant d'une part la diffusion multidirectionnelle des connaissances et d'autre part la création d'activités de mobilisation telles que : la participation des membres, la diffusion et la coproduction de connaissances à l'intérieur de l'équipe de recherche et en dehors du milieu universitaire pour la Nation abénakise et le Musée des Abénakis.

Les résultats des recherches entreprises par le Bureau du Ndakinna génèrent des données que les connaissances archéologiques viennent compléter afin d'obtenir un portrait de plus en plus représentatif de la présence abénakise au Québec. Cette perspective de collaboration entre différentes disciplines mobilise des connaissances de recherche multidisciplinaire (archéologie, environnement, anthropologie, etc.) et celles-ci sont non seulement destinées au milieu universitaire, mais aussi à la Nation abénakise. Elles ont le potentiel de produire des retombées tant sur les plans intellectuel et formatif que culturel, social et économique. 


\section{Retombées de l'approche collaborative et multidisciplinaire pour la Nation abénakise}

Du point de vue de la Nation Abénakise, ces recherches multidisciplinaires en collaboration avec les communautés nous aident à réfléchir et à formaliser notre conception du Ndakinna. Cela permet de la valoriser dans les différents processus de consultation et de gestion territoriale dans lesquels le Bureau du Ndakinna est appelé à nous conseiller et nous représenter. Pour le Bureau, cette démarche collaborative et multidisciplinaire alliant savoirs abénakis et savoirs scientifiques nous permet de mieux documenter les changements dans l'accès aux ressources et au territoire ainsi que les conséquences de ces transformations rapides sur le mode de vie de la Nation.

Le cas du lac Saint-Pierre, une vaste étendue d'eau au cœur du Ndakinna reconnue pour sa biodiversité remarquable et fréquentée depuis des temps immémoriaux par la Nation pour ses activités traditionnelles, est particulièrement éloquent en ce sens (figure 2). Les données (environnementales, qualitatives, archéologiques et cartographiques) recueillies dans le cadre d'une étude sur deux ans ont rendu possible une meilleure description des changements environnementaux relatifs aux développements agricoles, industriels, commerciaux et urbains accrus autour du lac. Cette recherche nous a permis de réaliser, à l'échelle régionale, la portée des effets présents et futurs de ce développement sur les droits abénakis en termes de pêche, de chasse, de trappe et de cueillette. De plus, elle a aidé à comprendre et à communiquer la nécessité de prendre des mesures pour remédier au déclin de l'écosystème du lac. 


\section{Figure 2}
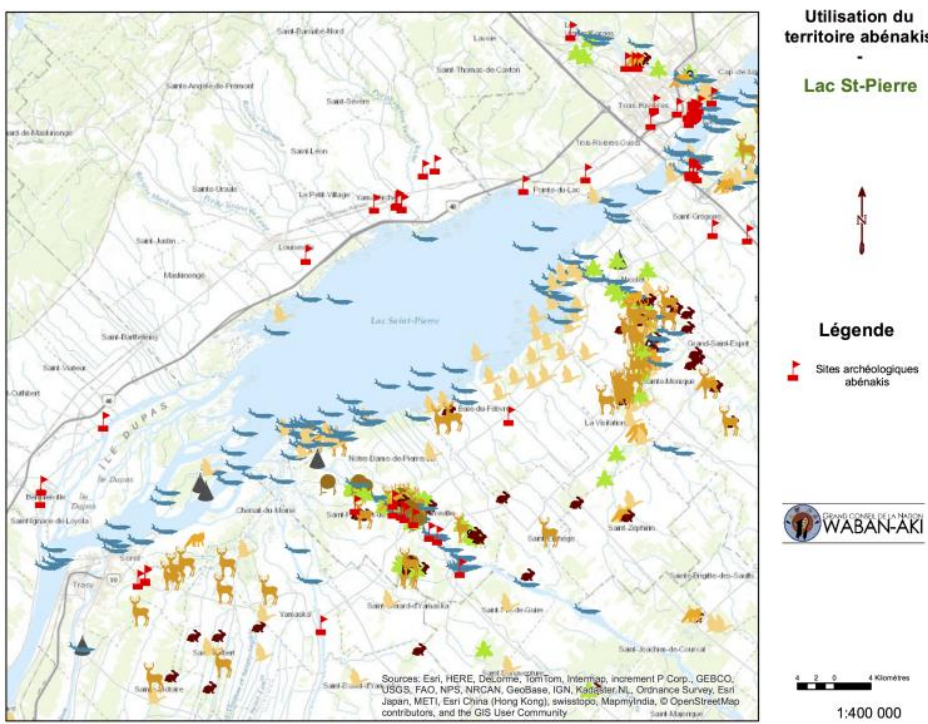

Aperçu de données multidisciplinaires sur la pêche recueillies dans le cadre d'une étude au sein de la Nation au lac Saint-Pierre. Ces données servent à mieux comprendre l'utilisation et l'occupation du Ndakinna. De plus, elles alimentent le processus d'affirmation territoriale et renforcent la capacité de la Nation à répondre adéquatement aux demandes de consultation sur son territoire.

(C) Les données d'habitat de la perchaude présentées sur la carte sont tirées de De la Chenelière et al. 2014 (CGCNWA.

Par ailleurs, cette étude a mené à l'émergence de résultats inattendus pertinents à notre compréhension de la territorialité abénakise contemporaine. Sachant que le lac est fortement anthropisé et ses berges se sont privatisées au fil des ans, nous n'anticipions pas que la Nation continue de se déplacer de manière régulière sur ce cours d'eau et ses affluents. Cette recherche a permis de valoriser et documenter les récits de déplacement de certains Abénakis et de retracer un réseau de circulation bien vivant, confirmé par les travaux 
archéologiques et historiques, où les croisements de rivières sont également propices à la découverte d'anciens campements.

Grâce à cette approche, nous jouons un rôle actif dans la production du savoir à propos du passé et du présent de la Nation et nous pouvons cerner les éléments de rupture et de continuité dans l'histoire du territoire. À l'échelle locale et régionale, cette démarche nous permet à notre tour de tisser des collaborations et nous aspirons de plus en plus à assumer un rôle d'intendance et exercer un leadership environnemental positif. À ce titre, et en raison de nos expertises variées, la Nation est présentement représentée au sein de divers organismes de concertation (notamment les tables de concertation régionales du lac Saint-Pierre et de l'estuaire fluvial) en plus d'être membre de deux équipes de rétablissement d'espèces en péril (celle des cyprins et petits percidés ainsi que celle du bar rayé). L'acquisition de ces connaissances multidisciplinaires par des méthodes collaboratives est centrale à l'affirmation de notre présence sur le territoire. Elle a mené également, en collaboration avec les Bureaux Environnement et Terre d'Odanak et de Wôlinak, à une résolution des Conseils d'Odanak et de Wôlinak appelant à une action rapide pour le rétablissement de la perchaude au lac Saint-Pierre.

\section{Les axes de déplacement sur le Ndakinna}

Les travaux archéologiques réalisés depuis 2009 ont généré un ensemble de données permettant de reconstituer en partie les axes de déplacement utilisés par les Abénakis. Ces axes de circulation ont été retranscrits à partir des sources historiques, des cartes anciennes, des sources orales et des découvertes archéologiques. Les résultats présentés sous forme de carte sont un outil visuel significatif qui explique la gestion du territoire et démontre l'utilisation des voies d'eau comme moyen de communication. Ce réseau hydrographique donnait accès aux différentes ressources naturelles et fauniques disponibles sur le territoire ainsi qu'au réseau est-ouest de la vallée du Saint-Laurent qui permettait de réaliser des échanges avec les 
Premières Nations situées au nord et à l'ouest de la vallée (figures 3 et 4).

Figure 3

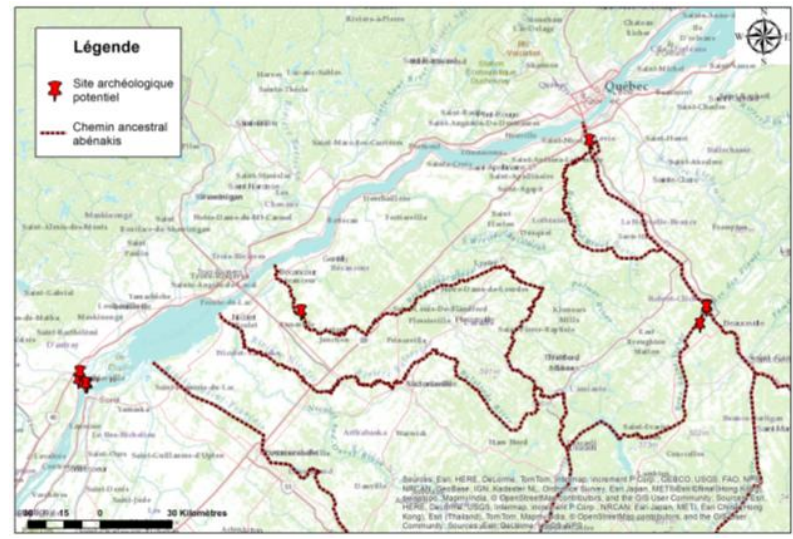

Carte des axes de déplacement reconstitués à partir des données archéologiques, des sources orales, des sources historiques et des cartes anciennes.

(c) GCNWA 


\section{Figure 4}

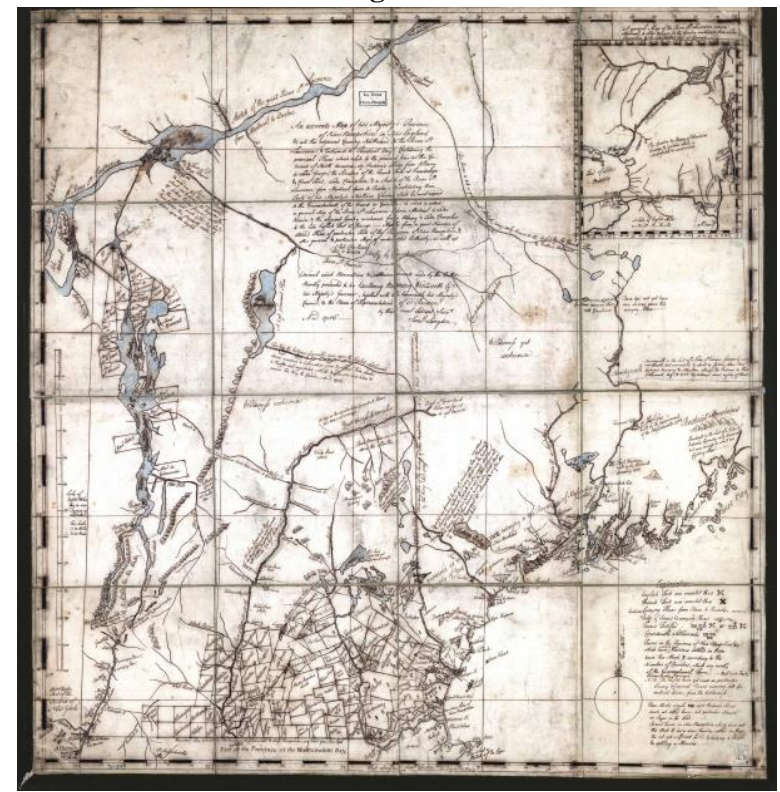

Carte de Samuel Langdon; Joseph Blanchard, 1756 Description manuscrite du sud de la Nouvelle-France incluant la rivière chaudière, Saint-Francois, Magog, avec les lacs, rivières et portages; état du Maine, New-Hampshire, Vermont, New-York.

(C) Vermont State Maps - On Line Database www.old-maps.com. Cette carte présente plusieurs portages empruntés par les Abénakis. Plusieurs informations provenant de cette carte ont servi à l'édition de la carte des axes de déplacement.

\section{Plan de formation et de mentorat}

L'équipe du Bureau du Ndakinna est composée de professionnels-chercheurs en début et milieu de carrière qui ont déjà une expérience significative en matière de formation d'étudiants et de 
stagiaires. Le partenariat et l'intégration de membres de communautés des Premières Nations à des projets de recherche sont de mises dans l'ensemble des programmes chapeautés par le GCNWA. Les étudiants, les stagiaires et les membres qui participent ont la possibilité d'avoir une formation multidisciplinaire théorique et pratique. Ils ont accès à un ensemble d'équipements, laboratoires, terrains et infrastructures diverses. Ils sont appelés à participer tant à des congrès scientifiques qu'à des présentations et à des formations publiques afin de discuter de l'avancée et des résultats des différents projets du Bureau du Ndakinna. La diffusion et le transfert de connaissance est un objectif important et permet de développer des aspects plus appliqués comme, en autres, la communication avec le public et les Membres des Premières Nations, qui garantissent une meilleure capacité d'intégration des étudiants dans divers milieux de travail.

Le musée des Abénakis (figure 5) joue un rôle important comme organisme responsable de la gestion des subventions, mais aussi comme acteur principal de la diffusion des résultats aux membres de la Nation abénakise et au public. Le Musée des Abénakis est le fer de lance des activités de la Société historique d'Odanak, un organisme à but non lucratif fondé en 1964 par le missionnaire Rémi Dolan dans le but d'assurer la promotion du développement culturel de la Nation abénakise et la conservation de ses traditions. " $L a$ mission du Musée est d'engager et de développer un dialogue viable et constructif entre la culture abénakise et les membres de la communauté, ainsi qu'entre la culture autochtone et les visiteurs $\gg^{1}$. Ce faisant, elle place au cœur des responsabilités du musée celle d'assurer la conservation et la transmission aux générations futures du savoir, du savoir-faire et des traditions de la Nation abénakise. En tant qu'outil au service des citoyens et visiteurs d'Odanak, de même que des habitants de la région du Centre-du-Québec, le Musée est à la fois un centre de diffusion de la culture abénakise, une voie d'accès pour les expositions autochtones venues de l'extérieur et un véhicule pour la présentation de projets non autochtones de la région. En assumant

1 Musée des Abénakis. museedesabenakis.ca/data/?page_id=161\&lang=fr 
ce triple rôle, le musée contribue de façon significative à la création d'une offre culturelle régionale originale et doit, par conséquent, travailler dans un esprit de partenariat avec les autres organismes culturels de la région. Présentés sous forme d'exposition, d'un Musée virtuel, d'un blogue et de capsules vidéo, les résultats des différents projets archéologiques sont diffusés et mis à jour au fur à mesure de l'avancement des travaux.

\section{Figure 5}

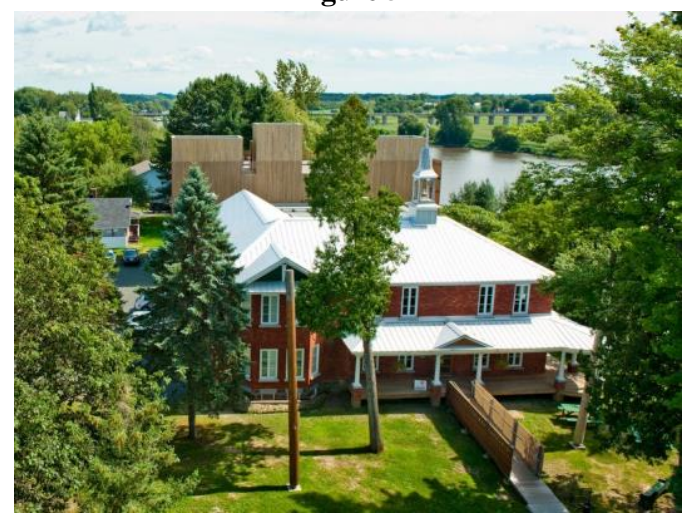

Le musée des Abénakis à Odanak situé dans le quadrilatère historique d'Odanak et à l'emplacement du site CaFe-7.

(C) Crédit Andre Gill, Société historique d'Odanak_9b Exposition permanente - Crédit Studio du ruisseau () SMQ

\section{Discussion, Les défis, l'influence et l'impact possible de la démarche}

La réussite d'une approche multidisciplinaire et collaborative repose en bonne partie sur le processus de préparation. C'est le moment où les liens de partenariat, d'échange et de confiance se tissent. Le succès de ce processus garantit en bonne partie la réussite 
du projet. Qu'ils soient d'ordre relationnel, logistique ou scientifique, les défis sont importants. Il est donc impératif de voir un tel projet sur le long terme. Les membres de l'équipe ont pris le temps d'échanger et de travailler avec les membres de la Nation à des projets tels que l'utilisation des ressources du lac Saint-Pierre, le Fort Odanak: le passé revisité, la Pointe du Moulin, Ulverton et l'Avenir des villages historiques Abénakis. Ils ont ainsi pu se sensibiliser, percevoir et connaître les différentes problématiques telles que la destruction de l'environnement, la disparition de certaines espèces animales, l'identification des sites archéologiques, l'érosion des sites côtiers, la mise en valeur des sites et des collections.

En ce moment, l'influence de notre collaboration prend plusieurs formes. La première concerne l'accessibilité pour la Nation abénakise à des partenariats entre le Bureau du Ndakinna et des spécialistes des institutions universitaires, des partenariats non seulement matériels mais qui reposent aussi sur le partage des connaissances entre les membres de la Nation abénakise et les unités de travail du Grand Conseil de la Nation Waban-Aki, dont le bureau du Ndakinna et ses différents partenaires. La seconde forme se situe au niveau de l'importance de la multidisciplinarité dans la compréhension des relations entre l'homme et son milieu ainsi que de l'impact de l'homme sur la nature et des conséquences sur les développements sociaux et technologiques de la Nation Waban-Aki. La troisième découle aussi de cette multidisciplinarité et situe l'archéologie dans un ensemble de connaissances qui permettent de faire sens du passé tout en collaborant à un projet collectif dans le présent, soit celui de l'affirmation territoriale, comme le relevaient McAnany et Rowe dans leur article paru en 2015 dans Journal of Field Archaeology (MCANANY \& ROWE, 2015).

Nous percevons deux défis importants dont il faudra gérer les

effets et les conséquences. Le premier concerne l'arrivée et la cohabitation à long terme des membres du Bureau et des partenaires dans une communauté. Un temps d'acclimatation est nécessaire à tous afin de bâtir et créer des échanges positifs. Le second est le clivage engendré par les résultats scientifiques et la perception du Ndakinna par les membres de la Nation. En effet, les interprétations scientifiques qu'elles soient archéologiques, environnementales, historiques ou anthropologiques peuvent être teintées soit d'une vue 
euro-canadienne ou encore religieuse et différer ainsi des idées et des traditions orales véhiculées à travers le temps par les Abénakis. Toutefois, les discussions générées par ce clivage entre les membres du Bureau, les partenaires et les membres de la Nation apportent une vision dynamique du territoire pour les générations futures.

Le mandat du Département est de répondre aux demandes de consultations acheminées par différents promoteurs ainsi que de contribuer à l'affirmation du territoire ancestral des Abénakis. Pour répondre à ce mandat, l'approche du Bureau du Ndakinna repose sur un partenariat multidisciplinaire et collaboratif permettant de mettre en relation l'ensemble des résultats. La recherche est une activité importante du Bureau. Les données environnementales, les données archéologiques, historiques et anthropologiques résultant des différentes études scientifiques réalisées et les connaissances acquises par les travaux d'enquête et de terrain effectués par les intervenants du Bureau du Ndakinna au GCNWA permettant d'acquérir les connaissances nécessaires à une gestion durable et responsable du territoire ancestral : le Ndakinna.

\section{Remerciements}

Nous tenons à remercier Hugo Mailhot Couture et Alex B. Perreault du GCNW pour l'aide à la production de cet article ainsi qu'aux membres de la Nation pour leur participation aux différents projets archéologiques mis en place depuis 2009. Il faut souligner le soutien permanent de l'équipe du Musée des Abénakis qui permet la réalisation des recherches archéologiques et la diffusion des résultats à la Nation abénakise et au public. Nos remerciements vont aussi au GCNWA et aux Conseils des Abénakis d'Odanak et de Wôlinak pour leurs appuis. 


\section{Bibliographie}

Assemblée des Premières Nations Québec-Labrador - APNQL, 2014 : Protocole de CALLOWAY, Colin G. The Western Abenakis of Vermont, 1600-1800. Norman: Les Presses de l'Université d'Oklahoma, 1994.

CHARLAND, Thomas-M. Histoire des Abénakis d'Odanak (16751937), Montréal: Éditions du Lévrier, 1964.

CLERMONT, N. 1980. Le contrat avec les animaux. Bestiaires sélectifs des Indiens nomades du Québec au moment du contact. Recherches amérindiennes au Québec, n. 10 (1-2) : 91-109.

DAY, Gordon M. Western Abenakis, IN Bruce G. TRIGGER (ed.), Handbook of North American Indians, n. 15 Northeast. Washington, D.C.: Smithsonian Institution, 1978, p. 148-159.

DE LA CHENELIÈRE, Veronik, BRODEUR Philippe \& MINGELBIER Marc, Restauration des habitats du lac Saint-Pierre: un prérequis au rétablissement de la perchaude. Le Naturaliste Canadien, n. 128, p.50-62, 2014.

MCANANY Patricia A., \& ROWE Sarah M., Re-visiting the field: Collaborative archaeology as paradigm shift. Journal of Field Archaeology, n. 40, p. 499-507, 2015.

SPECK, F. G., 1998 [1940; 1970]. Penobscot Man. Orono, Maine: University of Maine Press, 1998.

TREYVAUD, Geneviève \& PLOURDE Michel. Prospection archéologique, Odanak, 2010, Odanak, Musée des Abénakis, rapport déposé au ministère de la Culture et des Communications du Québec, 2010.

TREYVAUD, Geneviève \& PLOURDE Michel. Odanak, fouilles archéologiques 2011-2012, Odanak, Musée des Abénakis, rapport déposé au ministère de la Culture et des Communications du Québec, 2013. 
TREYVAUD, Geneviève \& PLOURDE Michel. Odanak, fouilles archéologiques 2013, Odanak, Musée des Abénakis, rapport déposé au ministère de la Culture et des Communications du Québec, 2014.

TREYVAUD, Geneviève \& PLOURDE Michel. Les Abénakis d'Odanak, un voyage archéologique. Odanak, Québec : La Société historique d'Odanak, 2017.

RECEBIDO EM: 01/10/2017

APROVADO EM: 10/12/2017 